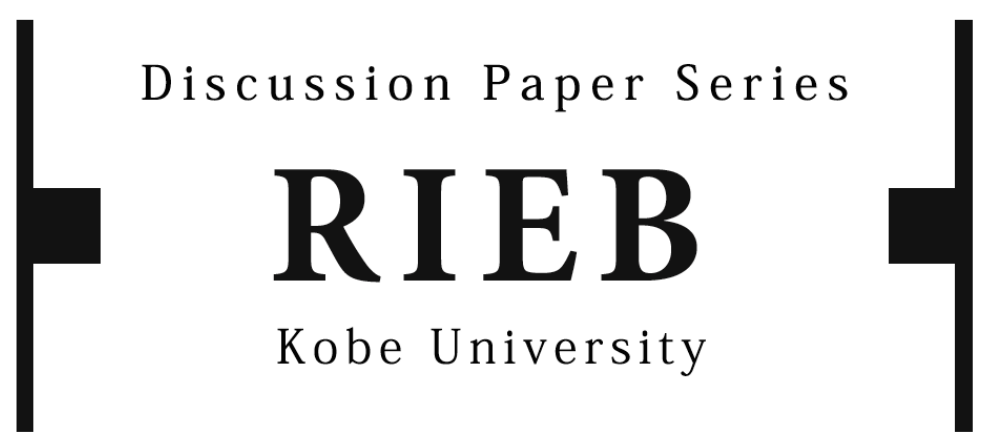

DP2012-20

Ergodic Chaos and Aggregate Stability: A Deterministic Discrete-Choice Model of Wealth Distribution Dynamics

Takashi KAMIHIGASHI

Revised October 20, 2012

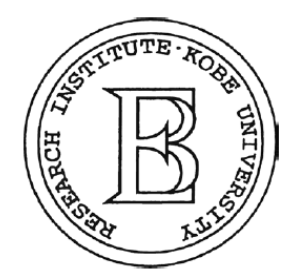

Research Institute for Economics and Business Administration Kobe University 


\title{
Ergodic Chaos and Aggregate Stability: A Deterministic Discrete-Choice Model of Wealth Distribution Dynamics*
}

\author{
Takashi Kamihigashi $^{\dagger}$
}

October 20, 2012

\begin{abstract}
This paper studies wealth distribution dynamics in a small open economy with a continuum of consumers indexed by initial wealth. Each of them solves a discrete-choice problem whose optimal policy function exhibits ergodic chaos. We show that for any initial distribution of wealth given by a density, the wealth distribution converges to a unique invariant distribution, and aggregate wealth converges to the corresponding value. Thus ergodic chaos leads to aggregate stability rather than instability. These results are illustrated with various numerical examples.
\end{abstract}

Keywords: Aggregation; ergodic chaos; stability; discrete choice; wealth distribution; Frobenius-Perron operator.

JEL Classification: C43, C60, D31

${ }^{*}$ Financial support from the Japan Economic Research Foundation and the Japan Society for the Promotion of Science is gratefully acknowledged.

${ }^{\dagger}$ RIEB, Kobe University, Rokkodai, Nada, Kobe 657-8501 JAPAN. Email: tkamihig@rieb.kobe-u.ac.jp. Tel/Fax: +81-78-803-7015. 


\section{Introduction}

It has been known since the mid-1980s that deterministic infinite-horizon optimization problems in economics can generate chaotic dynamics. Most of these problems are optimal growth models, which are typically solved using dynamic programming. ${ }^{1}$ Another class of models that exhibit chaos are discrete-choice problems of the type studied by Kamihigashi (2000a, 2000b, 2012), which give rise to complex dynamics in a very natural way.

Although many of these problems can be regarded as aggregate economies, they are in fact nothing more than single-agent optimization problems, none of which involves actual aggregation. A natural question then is, what if one aggregates the behavior of many agents each of whom obeys a chaotic policy?

To address this question in a simplest possible framework, we consider a small open economy with a continuum of consumers each of whom solves a special case of Kamihigashi's (2000a) discrete-choice problem. In this model the consumption good can only be purchased and consumed in whole units. We show that for any initial distribution of wealth given by a density, the wealth distribution converges to a unique invariant distribution, and aggregate wealth converges to the corresponding value. ${ }^{2}$ These results apply to cases in which initial wealth is almost identical across consumers. In such cases, a typical consumer's wealth and aggregate wealth follow similar paths at the beginning, but they differ completely in the long run: while the former keeps fluctuating forever, the latter converges to the unique long run value.

We use a simple discrete-choice model merely as an illustration of the idea that ergodic chaos may lead to aggregate stability rather than instability. This idea seems to be new in the context of aggregate economic dynamics though our results are immediate consequences of the "statistical stability" of the "Rény" map. Since the same property is shared by other maps such as the "tent" and "quadratic" maps (e.g., Lasota and Mackey, 1994), the idea of this paper can also be applied to an aggregated version of an optimal growth model whose optimal policy function is given by such a map.

The rest of the paper is organized as follows. In the next section we review the mathematical concepts used in our analysis. In Section 3 we present

\footnotetext{
${ }^{1}$ See Mitra et al. (2006) for a survey on chaos in optimal growth models. See Le Van and Dana (2003), Le Van (2006), and Kamihigashi (2008) for optimal growth and dynamic programming.

${ }^{2}$ See Bond et al. (2012) for wealth distribution dynamics in a two-country model.
} 
a special case of Kamihigashi's (2000a) discrete-choice problem along with some basic results. In Section 4 we show our results on the dynamics of the wealth distribution and aggregate wealth. These results are illustrated with numerical examples in Section 5.

\section{Preliminaries}

Here we review the mathematical concepts used in our analysis. Most of the definitions discussed here are adapted from Lasota and Mackey (1994).

Let $I$ denote an interval in $\mathbb{R}$ equipped with its Borel $\sigma$-algebra $\mathscr{B}$. For any (Borel-)measurable function $f: I \rightarrow \mathbb{R}$, we define $\|f\|=\int_{I}|f(x)| d x$. Let $L^{1}$ be the set of measurable functions $f: I \rightarrow \mathbb{R}$ such that $\|f\|<\infty$. Given any $\left\{f_{t}\right\}_{t=0}^{\infty} \subset L^{1}$ and $f^{*} \in L^{1}$, we write $\lim _{t \uparrow \infty} f_{t}=f^{*}$ if $\lim _{t \uparrow \infty}\left\|f_{t}-f^{*}\right\|=0$. Let $F$ be the set of probability densities on $I$, i.e., the set of nonnegative measurable functions $f: I \rightarrow \mathbb{R}_{+}$such that $\|f\|=1$. An operator $P: L^{1} \rightarrow$ $L^{1}$ is called a Markov operator if it is a linear operator and if $P f \in F$ for all $f \in F$. Given a Markov operator $P: L^{1} \rightarrow L^{1}$, a density $f \in F$ is called invariant under $P$ if $P f=f$. A Markov operator $P$ is called asymptotically stable if it has a unique invariant density $f^{*} \in F$ and if $\lim _{n \uparrow \infty} P^{n} f=f^{*}$ for all $f \in F$, where $P^{2} f=P(P f), P^{3} f=P\left(P^{2} f\right)$, etc. ${ }^{3}$

Let a measurable map $g: I \rightarrow I$ be given. For $B \in \mathscr{B}$, define

$$
g^{-1}(B)=\{x \in I: g(x) \in B\} .
$$

We say that $g$ is nonsingular if $\lambda\left(g^{-1}(B)\right)=0$ for any $B \in \mathscr{B}$ with $\lambda(B)=0$, where $\lambda$ is the Lebesgue measure on $I$. Provided that $g$ is nonsingular, the Frobenius-Perron operator $P_{g}: L^{1} \rightarrow L^{1}$ associated with $g$ is defined by

$$
\int_{B}\left(P_{g} f\right)(x) d x=\int_{g^{-1}(B)} f(x) d x, \quad B \in \mathscr{B} .
$$

This operator determines the evolution of densities under $g$. More specifically, if the distribution of a random variable $X$ is given by the density $f \in F$, then the distribution of $g(X)$ is given by the new density $P_{g} f \in F$. Since $P_{g}$ is linear by $(2.2)$, it follows that $P_{g}$ is a Markov operator. We say that $g$ is statistically stable if $P_{g}$ is asymptotically stable.

\footnotetext{
${ }^{3}$ To be precise, we require $f^{*}$ to be unique only up to sets of measure zero. Throughout this paper we follow the convention of identifying functions that differ only on a set of measure zero.
} 
A set $B \in \mathscr{B}$ is called invariant under $g$ if $g^{-1}(B)=B$. A density $f \in F$ is called invariant under $g$ if it is invariant under $P_{g}$. A density $f \in F$ invariant under $g$ is called ergodic if any set $B \in \mathscr{B}$ invariant under $g$ satisfies $\int_{B} f(x) d x=0$ or $\int_{B} f(x) d x=1$. Following Nishimura et al. (1994, Definition 1 ) we say that $g$ exhibits ergodic chaos if $g$ has an ergodic invariant density. Lasota and Mackey (1994, Proposition 5.6.2) show that if $g$ is statistically stable, then it exhibits ergodic chaos. ${ }^{4}$

\section{Individual Dynamics}

In this section we present an individual consumer's problem. It is a special case of the discrete-choice model studied by Kamihigashi (2000a). The problem here is a standard life-cycle model except that the consumption good can only be purchased and consumed in whole units. We assume that the price of the good is one, and the interest rate is constant over time. To be specific, consider the following maximization problem:

$$
\begin{aligned}
\max _{\left\{c_{t}, x_{t+1}\right\}_{t=0}^{\infty}} & \sum_{t=0}^{\infty} \beta^{t} u\left(c_{t}\right) \\
\text { s.t. } & x_{0} \geq 0 \text { given, } \\
\forall t \geq 0, & x_{t+1}=R x_{t}-c_{t}, \\
& x_{t+1} \geq 0, \\
& c_{t} \in \mathbb{Z}_{+},
\end{aligned}
$$

where $\beta \in(0,1)$ is the discount factor, $R>1$ is the gross interest rate, $x_{t+1}$ is wealth at the end of period $t$ (or $R x_{t}$ is wealth at the beginning of period $t$ ), and $c_{t}$ is consumption. The integer constraint (3.5) means that the consumption good must be purchased and consumed in whole units. We assume that the utility function $u: \mathbb{R}_{+} \rightarrow \mathbb{R}$ is continuous, concave, and strictly increasing with $u(0)=0$.

We say that a wealth path $\left\{x_{t}\right\}_{t=0}^{\infty}$ is optimal (from $x$ ) if there exists a consumption path $\left\{c_{t}\right\}_{t=0}^{\infty}$ such that $\left\{c_{t}, x_{t+1}\right\}$ solves the maximization problem (3.1)-(3.5) (with $x_{0}=x$ ). Given any subset $A$ of $\mathbb{R}_{+}$, a function $g: B \rightarrow \mathbb{R}_{+}$

\footnotetext{
${ }^{4}$ They show that if $g$ is statistically stable, then $g$ is "exact", which implies that $g$ is "mixing" and thus "ergodic"; see Lasota and Mackey (1994, p. 65, 66, 59). Their definition of ergodicity is equivalent to the definition of ergodic chaos above.
} 
is called an optimal policy function on $A$ if for any $x \in A$ there exists an optimal wealth path $\left\{x_{t}\right\}$ from $x$ such that $x_{1}=g(x)$. The following result is a special case of Kamihigashi (2000a, Theorem 1).

Lemma 3.1. Given any $y>0$, there exists $\bar{\beta}_{y} \in(0,1)$ such that if $\beta<\bar{\beta}_{y}$, then there exists a unique optimal policy function $g$ on $[0, y]$; furthermore, $g$ is given $b y^{5}$

$$
g(x)=R x \bmod 1
$$

See Kamihigashi (2000a) for an explanation and interpretation of this result. The map $g$ in (3.6) is a special case of a pseudo-random number number generator called a linear congruential generator. In fact the map $g$ itself typically generates erratic paths; see Figure $1 .^{6}$ For the rest of this paper we assume that $\beta<\bar{\beta}_{1}$, which implies that $g$ is a unique optimal policy function on $[0,1]$ by Lemma $3 \cdot 1 .^{7}$

Let $I=[0,1)$. Since $g$ maps $[0,1]$ into $I$, we restrict the domain of $g$ to $I$ unless otherwise stated. The following result is the basis for our analysis of wealth distribution dynamics in the next section.

Proposition 3.1. The map g is statistically stable and exhibits ergodic chaos.

Proof. Lasota and Mackey (1994, Theorem 6.2.1) show that the FrobeniusPerron operator $P_{g}$ associated with $g$ is asymptotically stable; thus $g$ is statically stable by definition, which implies that $g$ exhibits ergodic chaos; recall Section 2.

\section{Wealth Distribution Dynamics}

Consider a small open economy with a continuum of consumers indexed by initial wealth $x_{0}$. The price of the good and the gross interest rate are constant and exogenously given as in the previous section. Except for initial wealth, all consumers face the same problem (3.1)-(3.5), obeying the same optimal policy $g$. Let $F$ be the set of probability densities on $I$. We normalize

\footnotetext{
${ }^{5}$ For any $a, b \geq 0, a \bmod b=a-b \max \left\{n \in \mathbb{Z}_{+}: n b \leq a\right\}$.

${ }^{6}$ See Kamihigashi (2000a) and Gentle (2003) for more on random number generators. The map $g$ is known as the Rényi map, and is called the $R$-adic map if $R \in \mathbb{N}$ (Lasota and Mackey, 1994, p. 144).

${ }^{7}$ Even if $\beta$ does not satisfy this inequality, complex dynamics arise naturally in this model; see Kamihigashi (2000a, 2012).
} 


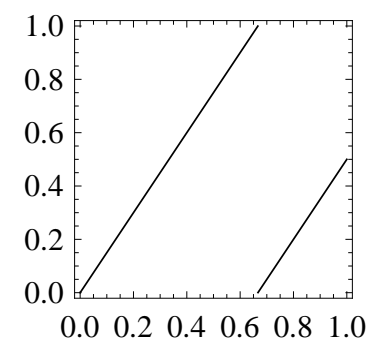

(a) $g(R=1.5)$

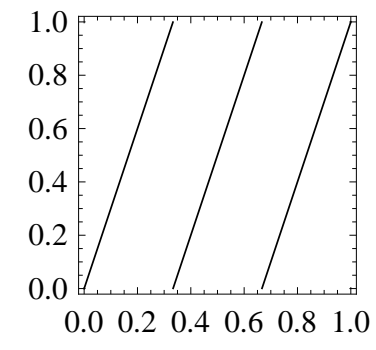

(c) $g(R=3)$

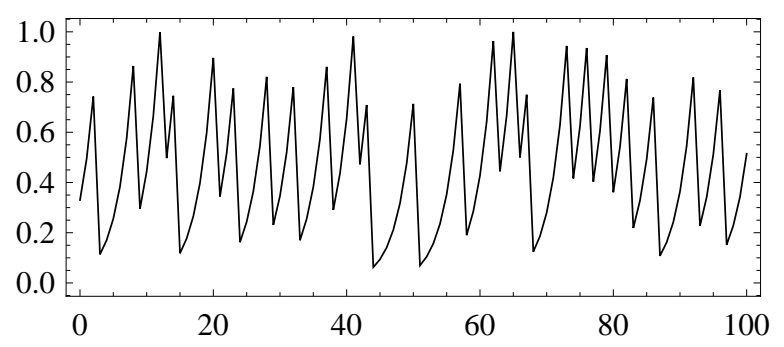

(b) $\left\{x_{t}\right\}\left(R=1.5, x_{0}=0.33\right)$

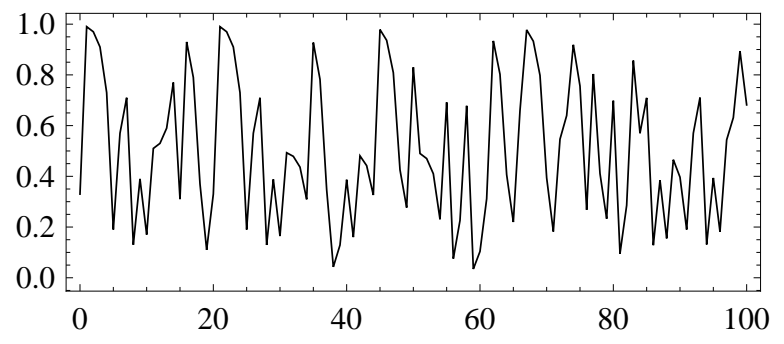

(d) $\left\{x_{t}\right\}\left(R=3, x_{0}=0.33\right)$

Figure 1: Optimal policy functions and optimal wealth paths $\left(\beta<\bar{\beta}_{1}\right)$

the population of consumers to unity, and assume that the distribution of initial wealth $x_{0}$ is given by a density $f_{0} \in F$. Let $f_{t} \in F$ denote the density of period- $t$ wealth $x_{t}$ for each $t \in \mathbb{Z}_{+}$. The evolution of these densities is determined by the Frobenius-Perron operator $P_{g}$ associated with $g$ (recall (2.2)); i.e., $f_{t+1}=P_{g} f_{t}$ for all $t \in \mathbb{Z}_{+}$. Aggregate wealth $X_{t}$ in period $t$ is given by

$$
X_{t}=\int_{0}^{1} x f_{t}(x) d x .
$$

We illustrate how various densities are mapped by $P_{g}$ after showing the following result.

Proposition 4.1. The map $g$ has a unique invariant density $f^{*} \in F$. Furthermore, for any $f_{0} \in F$, we have

$$
\begin{aligned}
\lim _{t \uparrow \infty} f_{t} & =f^{*} \\
\lim _{t \uparrow \infty} X_{t} & =\int_{0}^{1} x f^{*}(x) d x .
\end{aligned}
$$


Proof. Recall from the proof of Proposition 3.1 that $P_{g}$ is asymptotically stable; thus it has a unique invariant density $f^{*} \in F$, which satisfies (4.2). To see (4.3) note that for any $t \in \mathbb{Z}_{+}$,

$$
\begin{aligned}
\left|\int_{0}^{1} x f_{t}(x) d x-\int_{0}^{1} x f^{*}(x) d x\right| & \leq \int_{0}^{1} x\left|f_{t}(x)-f^{*}(x)\right| d x \\
& \leq \int_{0}^{1}\left|f_{t}(x)-f^{*}(x)\right| d x=\left\|f_{t}-f^{*}\right\| .
\end{aligned}
$$

Now (4.3) follows from (4.2).

Remark 4.1. Proposition 4.1 shows that even though individual dynamics may be extremely complicated, distributional and aggregate dynamics are always stable and convergent. Since (4.2) and (4.3) hold for any $f_{0} \in F$, they hold even if $f_{0}$ is almost concentrated at one point. For example, let $z \in(0,1)$ and $f_{0}=U_{z-\epsilon}^{z+\epsilon}$, where for any $a, b \in I$ with $a<b, U_{a}^{b}: I \rightarrow \mathbb{R}_{+}$ denotes the uniform distribution on $[a, b)$ :

$$
U_{a}^{b}(x)= \begin{cases}1 /(b-a) & \text { if } x \in[a, b) \\ 0 & \text { otherwise }\end{cases}
$$

Then for any $\epsilon>0$ with $z-\epsilon, z+\epsilon \in I$, (4.2) and (4.3) hold by Proposition 4.1. Thus erratic paths such as those in Figure 1 are impossible for aggregate wealth in the long run no matter how small $\epsilon>0$ may be.

Before we discuss Proposition 4.1 further, it is useful to understand how densities are mapped under $g$. For this purpose we express $P_{g}$ as

$$
\left(P_{g} f\right)(x)=\frac{1}{R}\left[\sum_{i \in \mathbb{N}: i \leq R} f\left(\frac{x+i-1}{R}\right)+f\left(\frac{x+[R]}{R}\right) \mathbb{1}\{x<g(1)\}\right],
$$

where $[R]$ is the largest integer $n$ with $n \leq R$; see Lasota and Mackey (1994, (6.2.4)) for the derivation of (4.7). Using this formula, we can explicitly compute $P_{g} f$ for any $f \in F$. Note that if $R \in \mathbb{N}$, then $g(1)=0$ and the second term in the square brackets in (4.7) is zero.

Figure 2 assumes that $R=3$ and $f_{0}=U_{a}^{b}$ with $0<a<1 / 3<b<2 / 3$. Panel (a) shows how $f_{0}$ is mapped under $g$. In panels (b) and (c), $f_{0}$ is decomposed into two parts:

$$
f_{0}=f_{0}^{1}+f_{0}^{2}, f_{0}^{1}(x)=\mathbb{1}_{[0,1 / 3)}(x) f_{0}(x), f_{0}^{2}(x)=\mathbb{1}_{[1 / 3,1)}(x) f_{0}(x),
$$




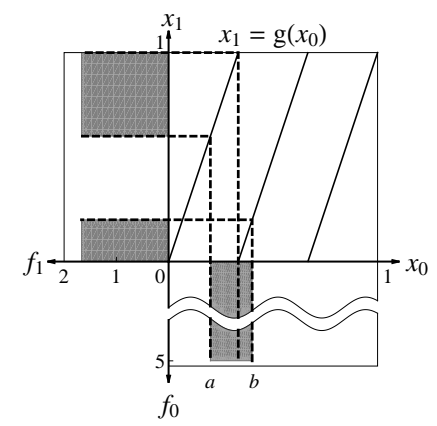

(a) $f_{1}=P_{g} f_{0}$

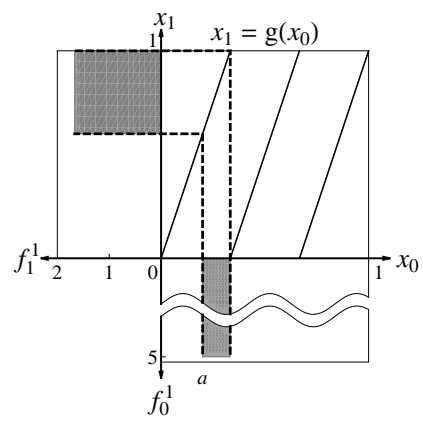

(b) $f_{1}^{1}=P_{g} f_{0}^{1}$

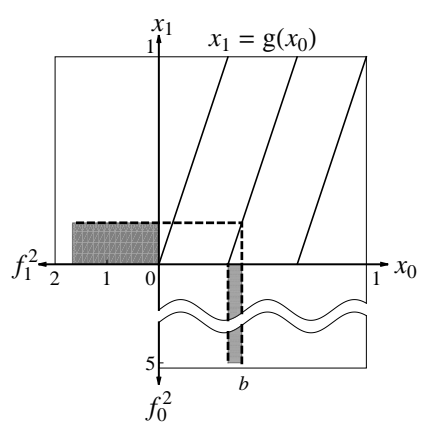

(c) $f_{1}^{2}=P_{g} f_{0}^{2}$

Figure 2: Decompositions of $f_{0}$ and $f_{1}$ as in (4.8) and (4.9) $(R=3)$

where $\mathbb{1}_{A}$ is the indicator function on set $A$; i.e., $\mathbb{1}_{A}(x)=1$ if $x \in A$, and $=0$ otherwise. The two components $f_{0}^{1}$ and $f_{0}^{2}$ are mapped separately under $g$ in panels (b) and (c). Since $P_{g}$ is a linear operator, we have

$$
f_{1}=P_{g} f_{0}=P_{g}\left(f_{0}^{1}+f_{0}^{2}\right)=P_{g} f_{0}^{1}+P_{g} f_{0}^{2}=f_{1}^{1}+f_{1}^{2},
$$

where $f_{1}^{i}=P_{g} f_{0}^{i}$ for $i=1,2$. Thus we can recover panel (a) by combining panels (b) and (c).

Figure 3 gives three more examples. In panel (a), $R=3$ and $f_{0}=U_{0}^{1}$. This density is separated by dashed lines into three components. Each of them has mass $1 / 3$ and is mapped to $(1 / 3) \mathbb{1}_{I}$. Multiplying this function by 3 yields $U_{0}^{1}$, which equals $f_{1}$ as well as $f_{0}$. Thus $U_{0}^{1}$ is an invariant density here.

In panel (b), $R=2.5$, but $f_{0}$ is the same as in panel (a). This density is separated by a dashed line into two components. The larger (left) component is mapped to a constant function on $I$, while the smaller to a constant function on $[0,1 / 2]$. Adding these functions results in $f_{1}$.

In panel (c), $R=2.5$, and $f_{0}(x)=2-2 x$. This density is divided into two components as in panel (b). The larger component is mapped to a decreasing linear function on $I$, while the smaller to a decreasing linear function on $[0,1 / 2]$. We obtain $f_{1}$ by adding these functions.

The convergence result (4.2) in Proposition 4.1 is particularly easy to understand if $R \in \mathbb{N}$. In this case, for any $n \in \mathbb{N}$, we have

$$
g^{n}(x)=R^{n} x \bmod 1 .
$$




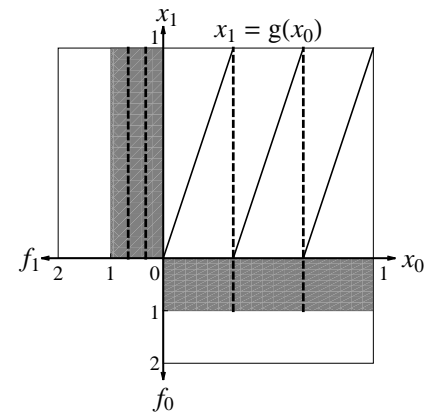

(a) $R=3$

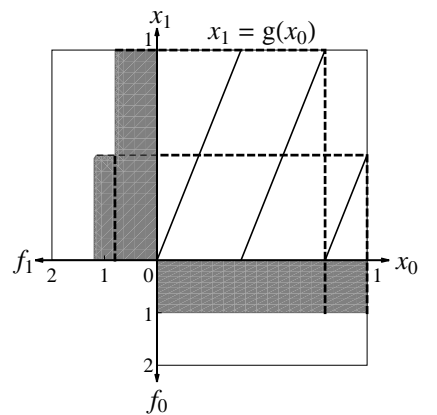

(b) $R=2.5$

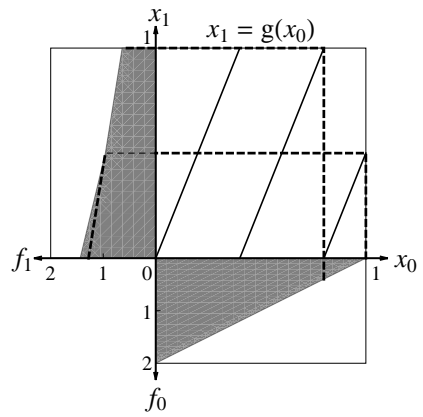

(c) $R=2.5$

Figure 3: Decompositions of $f_{0}$ and $f_{1}$

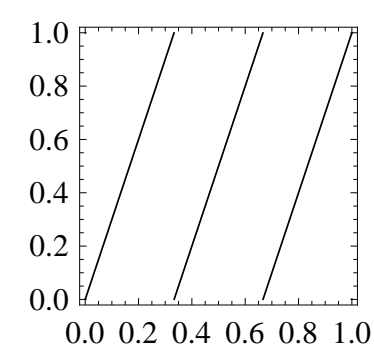

(a) $g(R=3)$

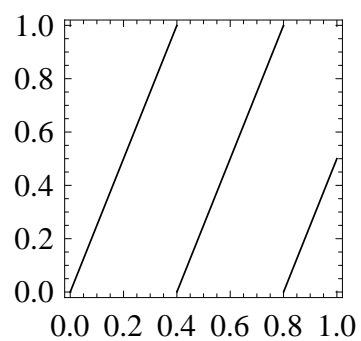

(d) $g(R=2.5)$

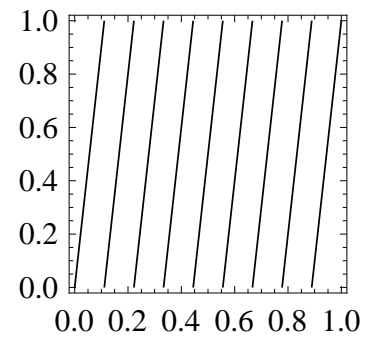

(b) $g^{2}(R=3)$

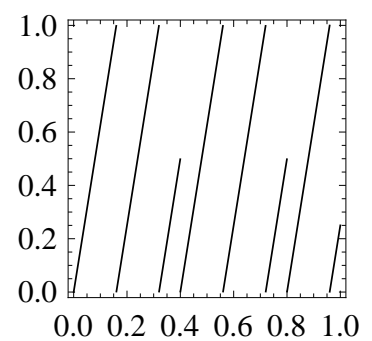

(e) $g^{2}(R=2.5)$

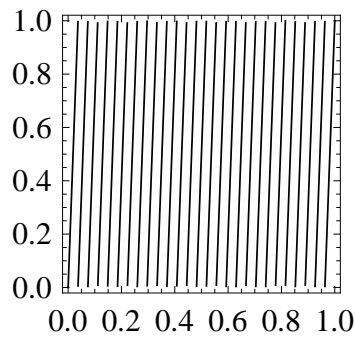

(c) $g^{3}(R=3)$

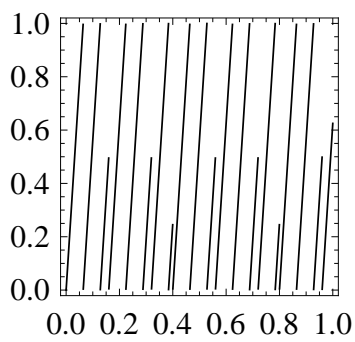

(f) $g^{3}(R=2.5)$

Figure 4: Graphs of $g, g^{2}$, and $g^{3}$ 
See Figure 4(a)-(c). Note that (4.10) is true only if $R \in \mathbb{N}$; see Figure $4(\mathrm{~d})-(\mathrm{f})$. For simplicity, let us assume for the moment that $f_{0}=U_{a}^{b}$ with $0 \leq a<b \leq 1$. Since $f_{t}=P_{g}^{t} f_{0}=P_{g^{t}} f_{0}$ for any $t \in \mathbb{N}$ (Lasota and Mackey, 1994, p. 43), we can visualize the transition from $f_{0}$ to $f_{t}$ in one step as in Figures 2 and 3. To this end, for any $t \in \mathbb{N}$, we divide $f_{0}$ into three components

$$
f_{0}^{t, 1}=\mathbb{1}_{[0, \tilde{a})} f_{0}, \quad f_{0}^{t, 2}=\mathbb{1}_{[\tilde{a}, \tilde{b})} f_{0}, \quad f_{0}^{t, 3}=\mathbb{1}_{[\tilde{b}, 1)} f_{0},
$$

where

$$
\begin{aligned}
& \tilde{a}=\min \left\{i / R^{t}: i / R^{t} \geq a, i \in \mathbb{Z}_{+}\right\}, \\
& \tilde{b}=\max \left\{i / R^{t}: i / R^{t} \leq b, i \in \mathbb{Z}_{+}\right\} .
\end{aligned}
$$

See Figure 5(a), where $R=3$ and $t=3$. Let $f_{t}^{i}=P_{g^{t}} f_{0}^{t, i}$ for $i=1,2,3$. Note that $f_{t}^{2}$ is a constant function on $I$; see Figure $5(\mathrm{c})$. As $t \uparrow \infty$, we have $\tilde{a} \rightarrow a$ and $\tilde{b} \rightarrow b$, and $f_{t}^{1}$ and $f_{t}^{3}$ converge to zero uniformly; see Figure $5(\mathrm{~b}),(\mathrm{d})$. Hence if $t$ is sufficiently large, $f_{t}$ approximately equals $f_{t}^{2}$, which in turn approximately equals $U_{0}^{1}$. This explains why $f_{t}$ converges to $f^{*}=U_{0}^{1}$ in the case $R \in \mathbb{N}$. If $R \notin \mathbb{N}$, however, we have $f^{*} \neq U_{0}^{1}$ :

Proposition 4.2. We have $f^{*}=U_{0}^{1}$ if and only if $R \in \mathbb{N}$. If $R \notin \mathbb{N}$, then $f^{*}$ is nonconstant, bounded, and decreasing (i.e., nonincreasing).

Proof. Suppose that $R \in \mathbb{N}$. Then it follows from Figure 3 (a) that $U_{0}^{1}$ is invariant. Since there is only one invariant density by Proposition 4.1, we have $f^{*}=U_{0}^{1}$. If $R \notin \mathbb{N}$, then $U_{0}^{1}$ cannot be invariant by Figure $3(\mathrm{~b})$, and $f^{*}$ is bounded and decreasing by Lasota and Yorke (1982, Theorem 4).

\section{$5 \quad$ Numerical Examples}

In this section we illustrate the results of the preceding section with a series of numerical examples. Throughout this section we assume that $f_{0}=U_{0.26}^{0.28}$; i.e., the initial distribution of wealth is almost concentrated at 0.27. All the examples here are computed by discretizing $I$ into 10,000 equally spaced grid points.

Figure 6 demonstrates that $\left\{f_{t}\right\}$ quickly converges to $U_{0}^{1}$ if $R=3$. As Proposition 4.2 shows, we have $f^{*}=U_{0}^{1}$ here. Figure 7 illustrates the stark 


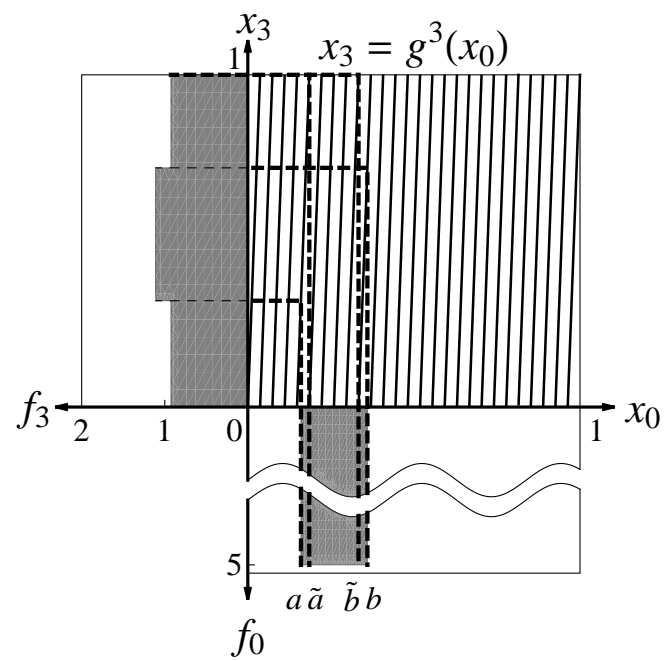

(a) $f_{3}=P_{g^{3}} f_{0}$

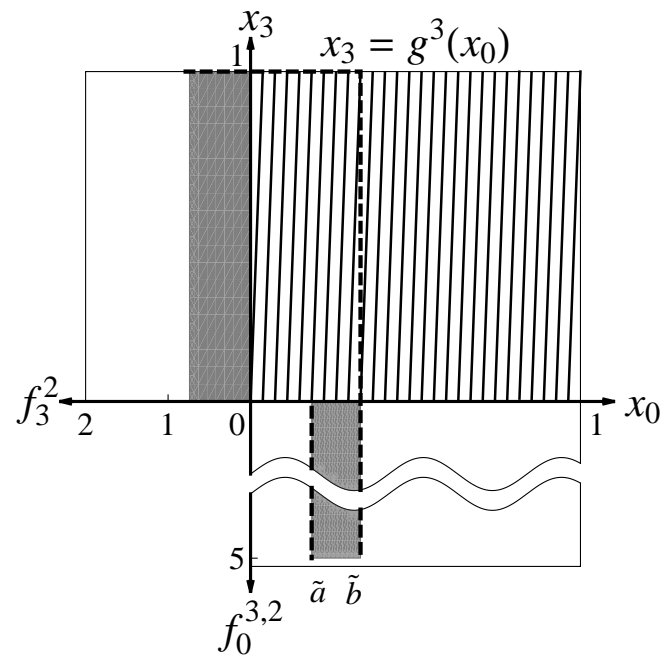

(c) $f_{3}^{2}=P_{g^{3}} f_{0}^{3,2}$

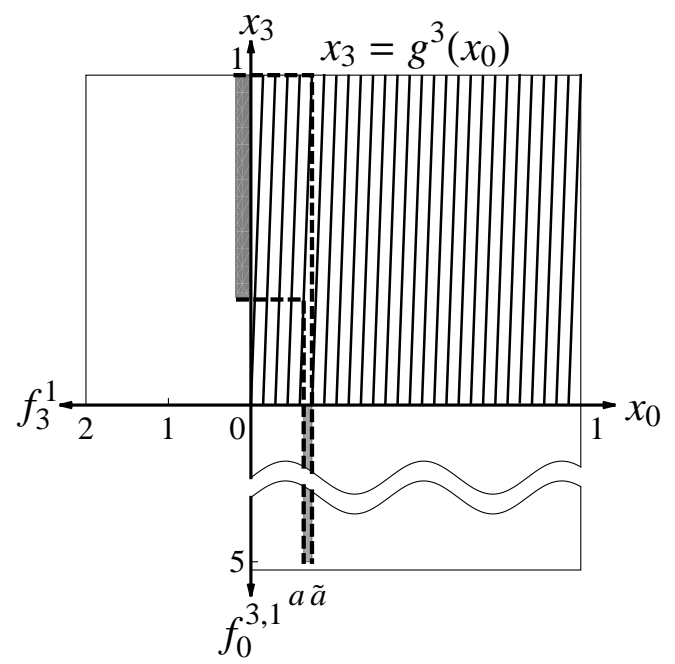

(b) $f_{3}^{1}=P_{g^{3}} f_{0}^{3,1}$

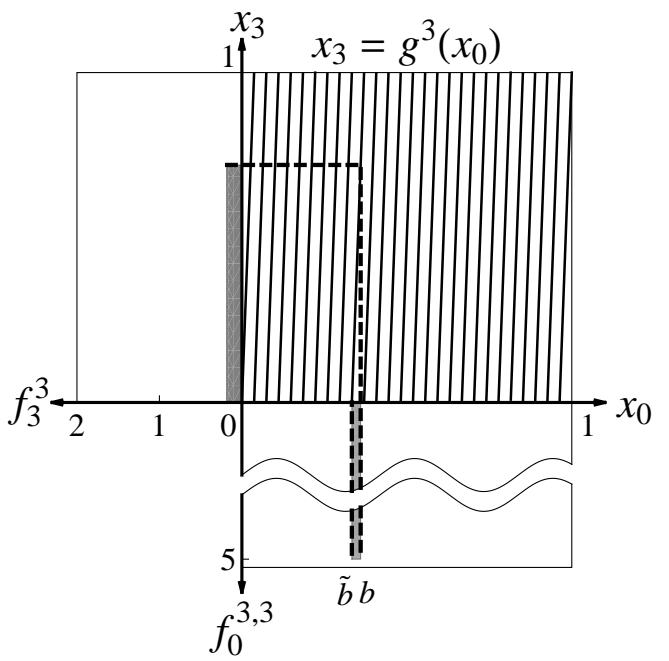

(d) $f_{3}^{3}=P_{g^{3}} f_{0}^{3,3}$

Figure 5: Decompositions of $f_{0}$ and $f_{3}\left(f_{0}=U_{a}^{b}, R=3\right)$ 

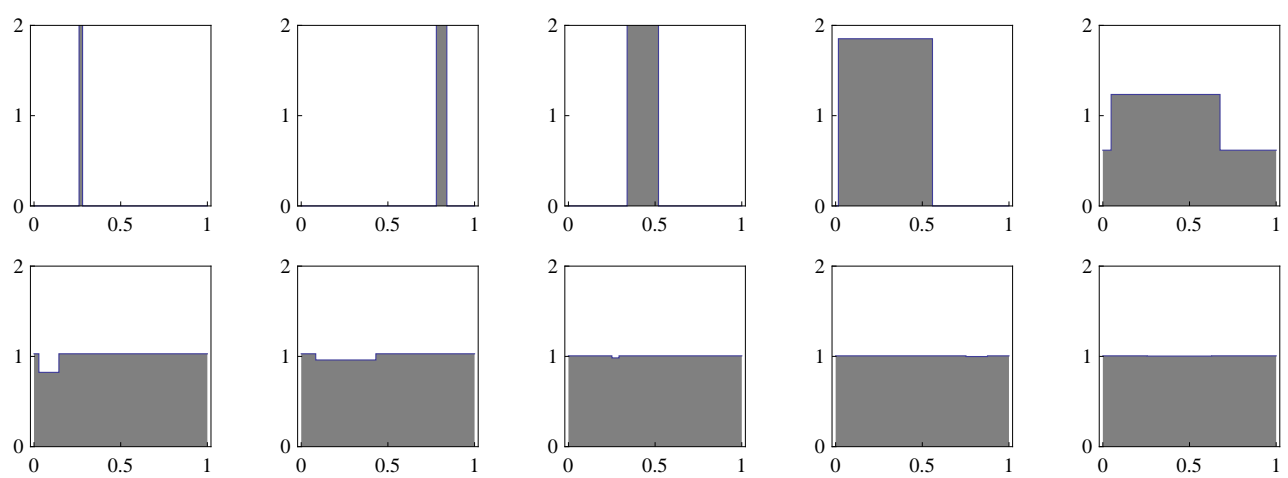

Figure 6: $f_{0}, f_{1}, \ldots, f_{9}(R=3)$
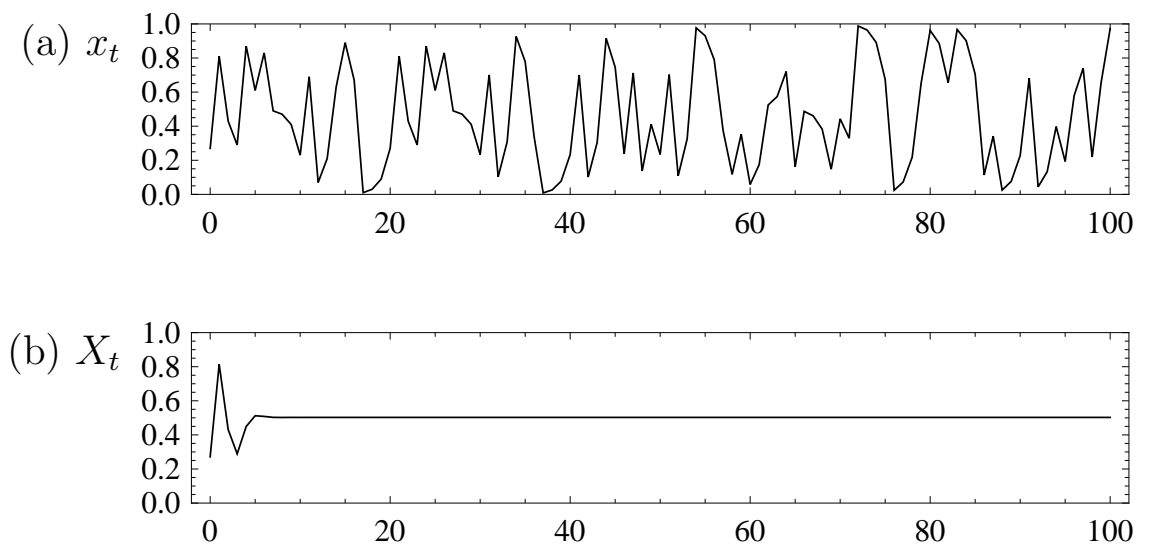

Figure 7: $\left\{x_{t}\right\}$ with $x_{0}=0.27$ and $\left\{X_{t}\right\}$ with $f_{0}=U_{0.26}^{0.28}(R=3)$ 

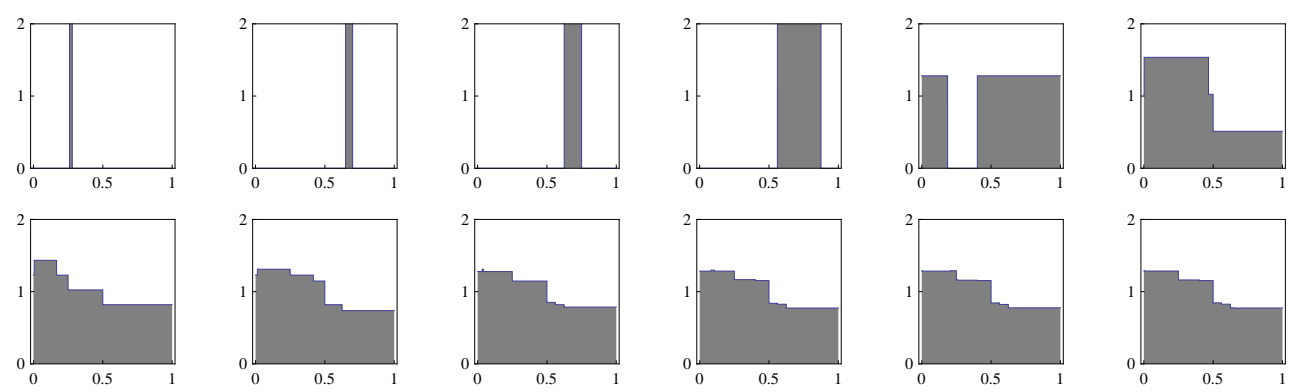

Figure 8: $f_{0}, f_{1}, \ldots, f_{11}(R=2.5)$

difference between individual and aggregate dynamics: while the individual wealth path $\left\{x_{t}\right\}$ with $x_{0}=0.27$ keeps fluctuating, the aggregate wealth path $\left\{X_{t}\right\}$ with $f_{0}=U_{0.26}^{0.28}$ converges very quickly.

Since $R$ is the "expansiveness" of the map $g$, we can expect that $\left\{f_{t}\right\}$ converges more slowly the smaller $R$ is. This can be seen by comparing Figure $6(R=3)$ and Figure $8(R=2.5)$. Figure 8 also confirms that $f^{*}$ is decreasing if $R \notin \mathbb{N}$, as shown by Proposition 4.2. Figure 9 shows that $\left\{X_{t}\right\}$ still converges fairly quickly, while $\left\{x_{t}\right\}$ keeps fluctuating. These points are further illustrated by Figures 10 and 11, where $R=1.5$.

If $R=1.1$, both $\left\{f_{t}\right\}$ and $\left\{X_{t}\right\}$ converge rather slowly; see Figures 12 and 13. Figure 12 confirms once again that $f^{*}$ is decreasing if $R \notin \mathbb{N}$, but shows that $f^{*}$ is considerably smoother here than in Figures 8 and 10. Figures 7 , 9,11 , and 13 suggest that the more erratic individual wealth is, the faster aggregate wealth converges.

\section{References}

Bond, E. W., K. Iwasa, and K. Nishimura (2012), "The dynamic HeckscherOhlin model: a diagrammatic approach," International Journal of Economic Theory 8, 197-211.

Gentle, J.E. (2003), Random Number Generation and Monte Carlo Methods, 2nd ed., New York: Springer.

Kamihigashi, T. (2000a), "The policy function of a discrete-choice problem is a random number generator," Japanese Economic Review 51, 51-71.

Kamihigashi, T. (2000b), "Indivisible labor implies chaos," Economic Theory $15,585-598$. 

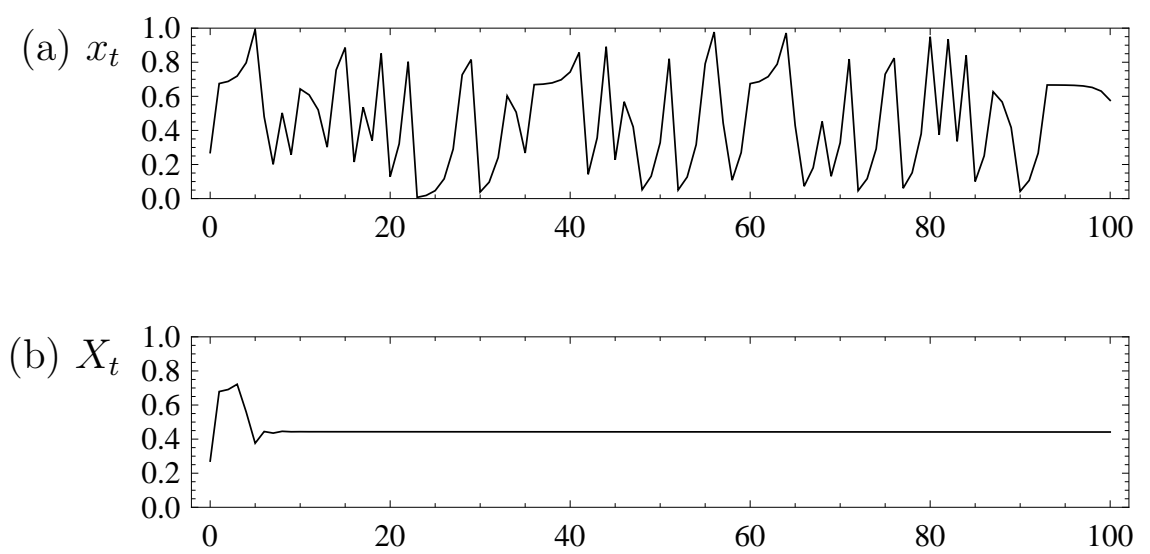

Figure 9: $\left\{x_{t}\right\}$ with $x_{0}=0.27$ and $\left\{X_{t}\right\}$ with $f_{0}=U_{0.26}^{0.28}(R=2.5)$
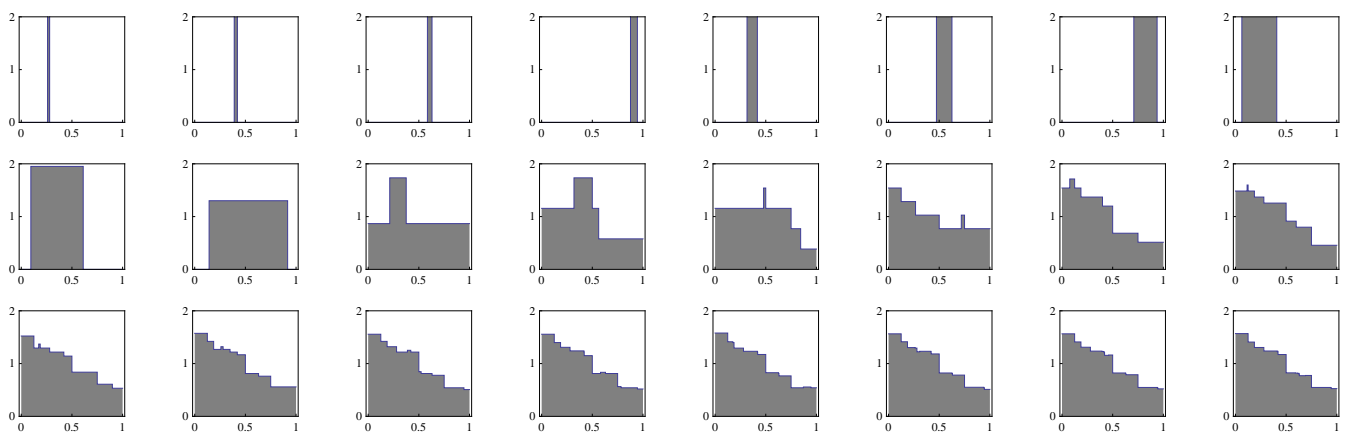

Figure 10: $f_{0}, f_{1}, \ldots, f_{23}(R=1.5)$
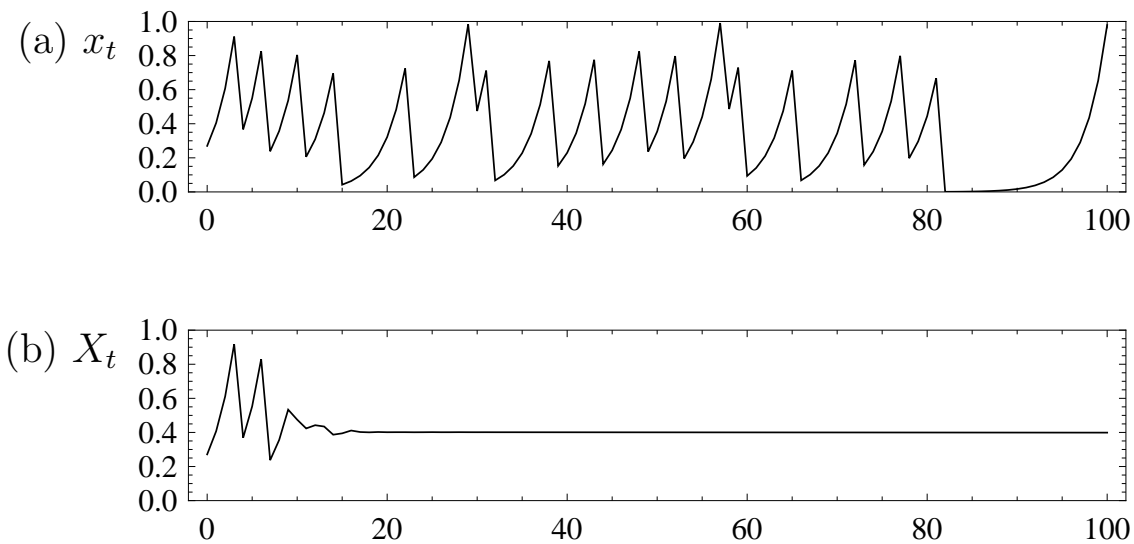

Figure 11: $\left\{x_{t}\right\}$ with $x_{0}=0.27$ and $\left\{X_{t}\right\}$ with $f_{0}=U_{0.26}^{0.28}(R=1.5)$ 


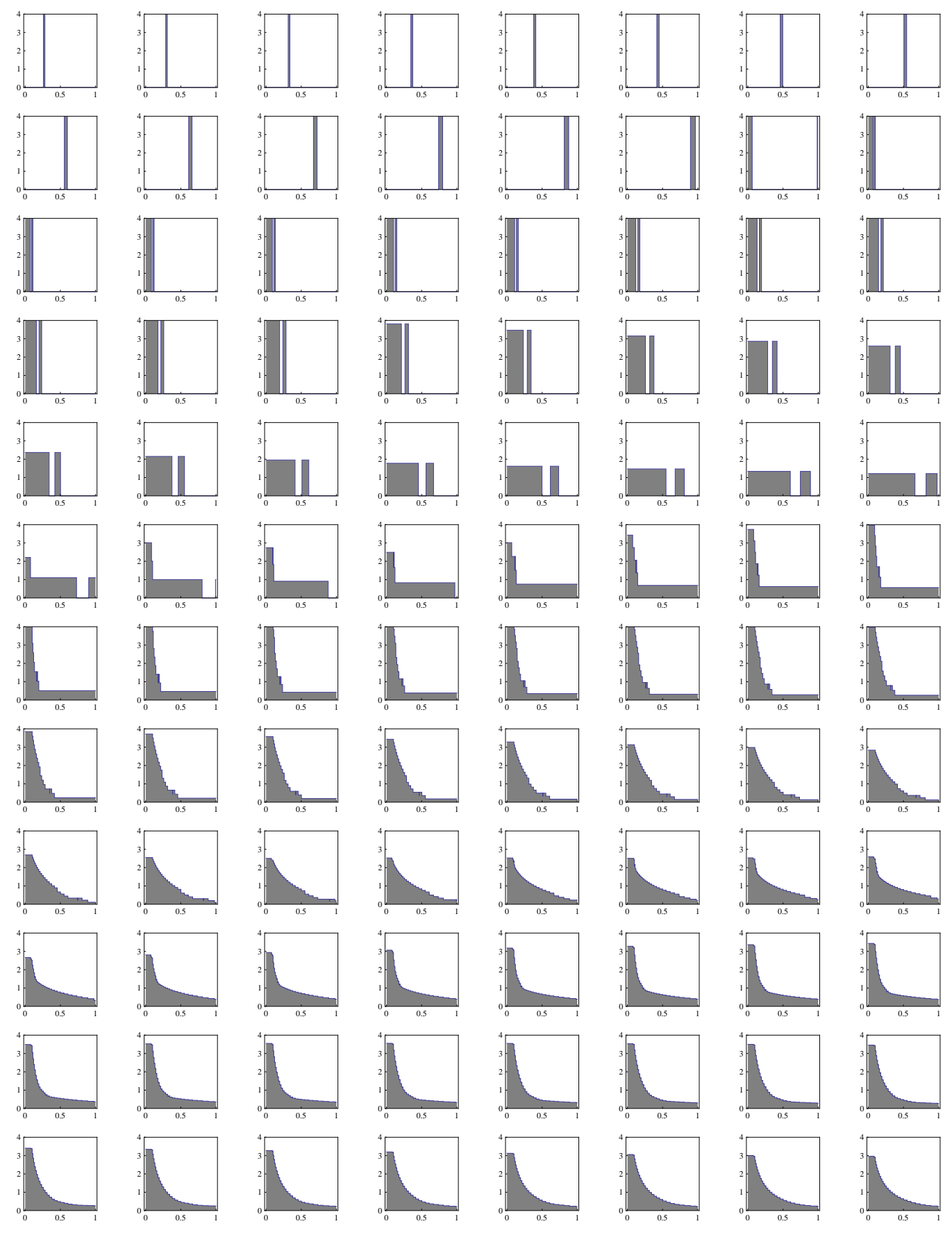

Figure 12: $f_{0}, f_{1}, \ldots, f_{95}(R=1.1)$ 

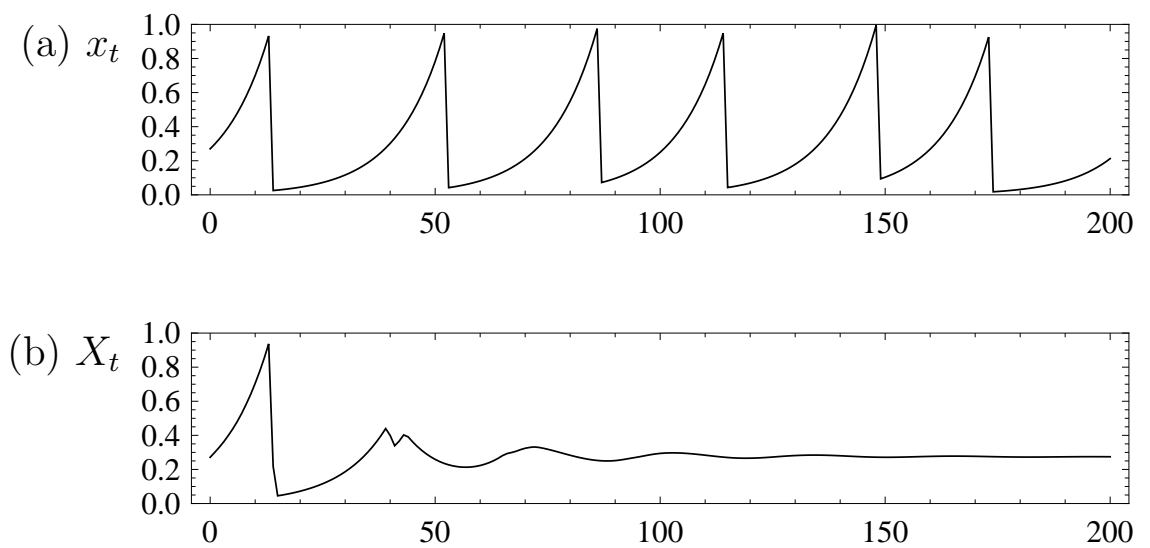

Figure 13: $\left\{x_{t}\right\}$ with $x_{0}=0.27$ and $\left\{X_{t}\right\}$ with $f_{0}=U_{0.26}^{0.28}(R=1.1)$

Kamihigashi, T. (2008), "On the principle of optimality for nonstationary deterministic dynamic programming," International Journal of Economic Theory 4, 519-525.

Kamihigashi, T. (2012), "Discrete choice and complex dynamics in deterministic optimization problems," Macroeconomic Dynamics 16, 52-69.

Lasota, A., and M. C. Mackey (1994), Chaos, Fractals, and Noise: Stochastic Aspects of Dynamics, 2nd ed., New York: Springer-Verlag.

Lasota, A., and J. A. Yorke, (1982), "Exact dynamical systems and the FrobeniusPerron operator," Transactions of the American Mathematical Society $273,375-384$.

Le Van, C. (2006), "Optimal growth models with discounted return," R.-A. Dana, C. Le Van, T. Mitra, and K. Nishimura, eds, Handbook on Optimal Growth 1: Discrete Time, pp. 19-54, Berlin: Springer.

Le Van, C., and R.-A. Dana (2003), Dynamic Programming in Economics, Dordrecht: Kluwer Academic Publishers.

Mitra, T., K. Nishimura, and G. Sorger (2006), "Optimal cycles and chaos," R.-A. Dana, C. Le Van, T. Mitra, and K. Nishimura, eds, Handbook on Optimal Growth 1: Discrete Time, pp. 141-169, Berlin: Springer.

Nishimura, K., G. Sorger, G., and M. Yano (1994), "Ergodic chaos in optimal growth models with low discount rates," Economic Theory 4, 705717 . 